$\xi_{p}=1$

\title{
Survey on Fault Detection and Diagnosis Using Neural Network in WBAN
}

\author{
${ }^{1}$ R.N.S.Kalpana , ${ }^{2}$ Dr.P.Nallathai \\ ${ }^{1}$ Asst.Professor, Department of Electronics and Communication engineering \\ TKR College of Engineering \& Technology, Meerpet, Saroor Nagar, Hyderabad. \\ E-mail: kalpana.mallapragada@gmail.com \\ ${ }^{2}$ Professor, Department of Electronics and Communication Engineering \\ TKR College of Engineering \& Technology, Meerpet, Saroor Nagar, Hyderabad. \\ *Corresponding author E-mail pnthai@ rediffmail.com
}

\begin{abstract}
:
Wireless Body Area Networks (WBAN) is the sensor network used for monitoring health information in e-health systems. WBAN is a combination of sensors used to obtain vital information from the body. It is a special type of WSN. WBAN technology should handle the data in a smart way by reacting to the monitored data and to evaluate the data. It requires fault detection and diagnosis methods for sensors used in WBAN. This paper gives a survey of different types of neural-network approaches for faults detection and diagnosis in WBAN using neural network.
\end{abstract}

Keywords: WBAN, Neural Network, fault diagnosis and fault detection

\section{Introduction:}

Nowadays Wireless sensor ne

twork has been used in health monitoring system to improve the quality of monitoring and presenting the vital information. Because of the less cost, low energy consumption and small in size, these sensors can be easily wearable or implanted in human body. This is a special type of WSN called Wireless body Area Network, used for monitoring the body conditions.

The complex healthcare equipments and wired equipments to monitor patient's vital statistics can be replaced by this WBAN [1]. WBAN should provide real time feedback without disturbing the patient's comfort [2]. The data obtained from WBAN should provide the doctor about the patient's vital information.

For the better real time feedback, the WBAN should have more accurate fault detection and diagnosis method to avoid misinterpretation of data from the human body. The misinterpretation of data leads to the patient into fatal condition. Fault is a type of threat, which is a hardware or software defect. There are two types of fault; active and passive. If the fault can easily be noticed then it is active fault. Example for active fault is dead battery of sensor [3]. If a fault in the software program, then it is passive fault. This paper gives the survey about fault detection and diagnosis in WBAN using neural network.

\section{Health Monitoring System}

A health monitoring system is having many sensors nodes which measures and reports patient's health and physiological state to the doctor. The sensors can be placed on patient's body. The sensor type defines the location of the sensor on human body. The sensors can be worn as standalone device or built in ornament. WBAN can also be used in health care for the recording of ECG,EEG,EMG [4]. WBAN is a combination of sensors, actuators and process control element. It consists of different types of sensor nodes for monitoring and provide response to the information obtained from the sensors, through wireless communication. Sensors are to monitor the patient's body and actuators for administering medicine to patient when required situation occurred guided by doctor, process control devices for establishing communication between sensors and actuators. The block diagram for real time health monitoring system is shown in figure. 1.

Sensors: Sensors are small electronic devices which are designed for converting physical or chemical information to analog or digital signals. Sensors supply the input to the feedback control systems. The sensor nodes can be placed on either on the body or under the skin of a patient to monitor the parameters of human body such as ECG, EEG, body movement, temperature, blood pressure, etc . If the sensor is placed on the surface of the body, then it is called Body surface sensor. If the sensor is placed under the skin or inside the 
tissue body then it is called implant sensor. If the sensor is not in contact with the body then it is called external sensor.

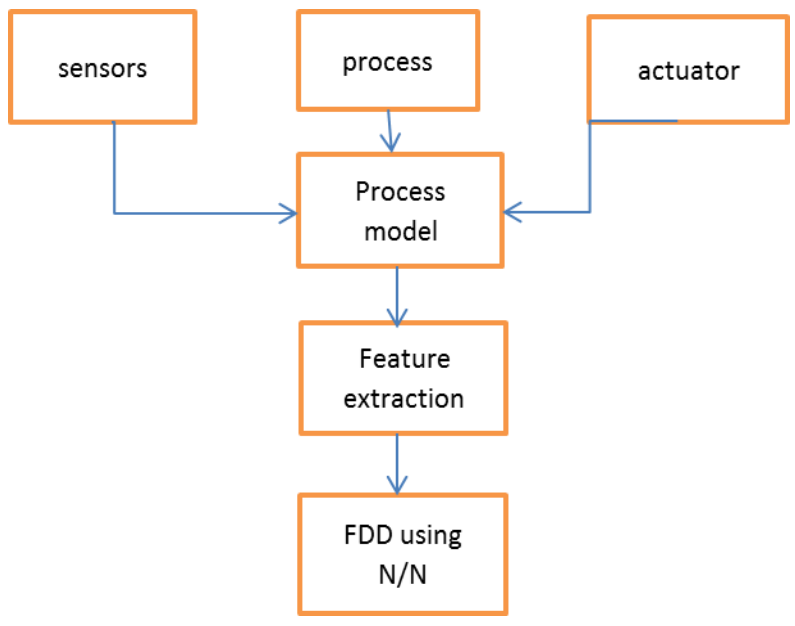

Figure 1 Block diagram for health monitoring system

Actuators: Actuators are devices which will act on the information obtained from the sensors based on the programmed instructions. In case of diabetic patients, actuators will administer proper doses of hypoglycaemic agent to support glucose level when there is a change in the glucose level of the patient. Depends upon the feedback signals received from the control unit, the actuator will give the response. For example audio feedback devices are used in the position monitoring to intimate the improper performance warning risk postures [5], gait diagnosis [6], and visual feedback devices are used for the illustration of current performance and desired objectives (e.g. 3D shoulder position detection [5]

Control functions: Control system or Process control consist of data processing and control algorithm. Accuracy level and control variable recorded from the sensors, processing speed will affect the performance of the system [7]. There are two different types of control based modes 1. Knowledge based control method example if fuzzy logic in which control rules are given linguisticalluy.2. Model based control method, which is divided into PID controller which can take strategies like Self-adaptive Control, Stochastic Control Model Predictive Control (MPC), and Robust Control.

WBAN Architecture: Real time health monitoring system contains tiny sensors for measuring the vital signals from the human body and a wireless sensor network for the communication between the sensors and the actuators, which are used for dispensing pills. The architecture of the sensor network of WBAN play an important role for continuous monitoring of patients. There are three types of architectures which are integrated mode, intra-BAN mode, and interBAN mode [8].

Integrated BAN mode: In this architecture, sensors, actuators and a controller are placed in a single node. A single node consists of several sensors and a controller unit housed together to collect and process data from the patient [9]. The advantage of this method is the fast feedback delivery and response time, but it costs very high power consumption.

Intra-BAN mode: In this architecture, the sensors, actuators and control unit are placed in independent nodes. The control unit collects all information from the sensors and sends feedbacks to actuators. The advantage of this mode is unnecessary distractions are avoided by providing different nodes for sensors and actuators, this causes the reduction in complexity and power consumption.
Inter-BAN mode : In this architecture an access point will be used for the incoming sensing data and outgoing feedback commands [10] on human body through a computer control centre or the human body respectively. An inter-BAN prototype of a modern healthcare system which involves the use of sensors (glucose level monitor, body temperature detection, etc.), a base station (PC or mobile phones), Internet, and a web browser is explained in [10]. This system is able to gather feedback from doctors and professions in distance. Use of processing control functions will reduce the complexity of control algorithms and correspondingly provide most accurate feedback information.

\section{Fault Detection Methods Using Neural Network}

For a better WBAN, the network should be fault tolerant and reliable. Fault can be defined as malfunctions of system failure which affects the performance of the system. It may be hardware failure or software failure. Faults should be noticed then and there to avoid the malfunctioning of the system. If the system works without any deterioration from its performance in the presence of fault is called fault tolerance. For the proper working of the system the fault should be detected, diagnosed and removed from the system. A fault tolerant system can be achieved by using two methods namely fault masking and fault isolation. The first step in any fault tolerant system is fault detection. Fault detection can be classified into online or offline detection.

Offline detection: For any wireless or wired network, a detection unit is necessary to find the unexpected error in the network. In this offline detection, detection phase is enabled during the idle period of the network.

Online detection: It is a real time procedure adopted mainly in wireless network. It is categorized into two types; explicit detection and implicit detection.

Explicit detection: This fault detection technique is application specific. When the event is detected, it will send an alarm. The fault detection methods for WSN will not be suitable for WBAN, since WBAN consist of many tiny heterogeneous sensors used in various environments. The anomaly detection and the malfunctioning of the sensors may be accidental or intentional based on the environment condition.

Implicit detection: It require the knowledge about the network environment to detect faults. Implicit detection is again divided into two types.

Active model: In this model, each node in the network used to send message continuously in the network used to send message continuously to the controller to maintain their live status. If the controller didn't receive any message from nodes for some period of time, then it detects that the node is dead[3].

Passive model: In this model, the controller will receive an alarm whenever fault occur in the node. This type of detection save the energy.

\section{Fault Detection Based on ANN}

The science of designing intelligent machine is referred as machine learning and the tools developed for this purposes are called as neural networks. The sensors in WBAN are prone to many other anomalies resulting bad sensor connection, energy depletion, leading unexpected results and faulty diagnosis and cause of critical health situation. In Anomaly detection, the significance feature of the ANN is that it will interpret the signal out come and analyse through data space [11]. Conjugate gradient descent algorithm for getting 
regression associative memory for training and prediction has been proposed in this paper. By utilizing learning based NN for WBAN can helps the system to improve the efficiency and signal prediction. ANN based learning can locate two faulty sensors signals with recovery of drift failure and bidirectional data associative memory with temporal coding is use for FPGA implementation for interconnections. In [11], gradient descent node training for MLP has been replaced by Non Linear Principle Component Analysis(NLCPCA), Machine Learning Applications to WBAN uses pattern recognition from unsupervised MPCA(Multi Linear Principle Component Analysis) to supervised regression method. It is difficult to provide optimal solution for the nonlinear optimization for a system with max throughput. This context consists of pre-processing stage with which uses component analysis nonlinear principle method for dimensionality reduction. Optimization method based on conjugate gradient for training phase ANN which automatically generate the desired training samples.

According to the study of [12], the performance analysis of the SVM, K-NN, BPNN, for motor imaginary into right hand movement and left hand movement specifies BPNN is best classifier has got good error reduction. The signal has been carried out using DWT. The features were fed to SVM, K-NN with five cross validation and they used supervised learning. BPNN which is machine learning algorithms and it is second classifier [13]. Anew fault detection method using an adaptive $\mathrm{NN}$ has been derived RBF, which has been used as fault classifier.

Anomaly detection algorithm for medical wireless sensor network is proposed in [14]. In this method, a classification algorithm has been used to detect abnormal values of the patient. A record will be maintained to store the normal values and the obtained values will be compared with this record and the abnormality will be detected. In [14], J48 decision tree algorithm, a linear regression based abnormal detection is discussed. This algorithm is used to predict the abnormalities present in the sensors as well as in the network. It consists of two phases; training and detection. Training phase will generate a classification model to classify the abnormal data from the normal data. In detection phase the abnormal data are identified based on the deviation from the normal data. After finding the abnormal value, regression coefficients are used to predict the value. If more than two anomalies are detected, then alarm will be given to alert the doctor.

In [15], the authors worked on an algorithm for identifying faulty sensor using minima and maxima values of y parameters. In [16], k-nearest Neighbour algorithm has been used to detect outliers of the faulty sensors based on KNN distance. This system is based on the detection of deviation of measurement from referred measurement time series. The authors analysed four methods of fault detection, like, rule based fault detection, estimation based fault detection, time series based fault detection and learning based fault detection. Auto regression is not suitable for anomaly detection. So Support vector machine SVM, Naïve Bayes, Baysian network are used for anomaly detection for multidimensional data.

In [17], the Probabilistic Neural Network (PNN) was used for temperature sensor fault detection. In this PNN, the learning process stores the sample data in the network itself. The samples will be classified based on the similarity. This PNN was able to detect the faulty sensors.

In [18], a type of neural network called Time delay Neural Network (TDNN), was used for the detection of hardware fault. It is a feed forward and time shift invariant network. The hardware fault detection using TDNN is performed by three different tasks. The first task is to classify the sample, next is to detect the fault sample and the third task is to control the program. The disadvantage is the length of window which should be static used for the detection.

The disadvantage of the TDNN can be overcome by Locally Recurrent Neural Network (LRNN) [19]. The general structure of LRNN contains three layers. The first layer and the third layer is a combination of static neurons and non linear output units. The middle layer contains dynamic neurons and non linear outputs. This LRNN can deal dynamic window size for detection with time series patterns.

Fault diagnosis: Fault diagnosis is the process to identify the isolated faults based on the characteristics and behavior of the network. From suspicious nodes and from irrelevant triggers faulty nodes should be distinguished. This can be achieved by applying previous data and sharing knowledge. The faults should be managed at the element level or in the communication link level.

Fault diagnosis based on Fault Tree Analysis: According to the study from [20], Fault Tree Analysis (FTA) method was used for the interpretation of the fault from the system. FTA analysed the cause for the system failure and reasons. Based on the analysis of the fault which can be eliminated from the system. In FTA, the system was divided into three node levels; the end node level 'leaves', the middle node level 'branch', and the top node level 'root'. These node levels were analysed separately in the bottom -up propagation direction. In [21], the authors proposed a top-down FTA method. In this method a Boolean relationship between the nodes were analysed and the faults removed.

Fault diagnosis based on expert system: An Expert system utilizes the decision making property of human brain. In [22] an expert system is developed to solve, complicated problem by reasoning ability will be used for the fault diagnosis from the facts and rules.

The components present in an expert system are knowledge base and interference reasoning. The step involve in an expert system are the classification of reason for the failure of the system and the solution to restore the system for normal operation. A neural network is used to diagnosis the faults in the system and expert system confirms the diagnosis or provide another solution,

In [23] fault tree analysis and neural network can used to construct FD expert system. In this method interference based fault tree and knowledge based neural network have been implemented for fault diagnosis.

In order to maintain the operation, to avoid damages or accidents, the automatic control system serves to indicate undesired process data. The traditional methods like signal based method and analytical method are limited to efficiency. The expert system is complex logical process demanding intelligent decision.

Fault diagnosis based on Bayesian network: The Bayesian theory is the theory of reasoning. Bayesian theorem computes the possibility for related event to happen. Bayesian theory provides very efficient methodology to deduce inference from observation. The Bayesian perspective is applicable to $\mathrm{NN}$ model is called Baysian network [24].

The major advantage of Bayesian network over a back propagation is the learning process is well defined, avoiding the need of may learning constraints like gain stop on, cross validation, Training set separation

The NAIVE Bayesian network is uses linear network. There are many variant in naïve Bayesian network tan [tree augmented naïve] 
which uses MDN as classifier. Iterative NAIVE base and difference boosting neural network.

In [25], on an ovulated system if the output of a system is satisfied the by the definition of the system then it can called as the NOC condition (normal operating condition), if that failed it is called as fault. This monitoring is called as the FDD (fault detection and diagnosis). There are two classes of FDD 1.model based FDD 2. Database based FDD. Model based FDD exploits the mathematical model, which is very effective. Data driven model is (signal based or statistical based). It doesn't exploit the physical data of system but it will gate data from the sensors or actuator. Here it is exiling the data driven FDD and BASYEAN network, which is the classical practice of the statistical method.

Under the multi-dimensional hypothesis of the NOC. If the fault is detected, supervised classification methods can be employed. In order to classify the new observation is known class construction classifier is required. In supervised learning no of classes datum of trading data is required. [24]

Conditional Gaussian network Bayesian network the integration of NOC class and different faults of Bayesian system can be used for CGN classifier for computation of the probabilistic limits guarantee the false alarm rate. It allows increasing the classification accuracy.

\section{Conclusion}

The objective of this review paper is to analyze the role of the neural network in the process of fault diagnosis and detection in WBAN. In this paper we explained the neural network types used for fault diagnosis and detection. This survey explains the strong and weak point of various types of neural networks used for fault diagnosis and detection. This paper describes the comprehensive issue present in the fault diagnosis and detection using neural network in WBAN technology.

\section{References}

[1] F. Akyildiz, Weilian Su, Yogesh Sankara subramaniam, and Erdal Cayirci.: A survey on sensor networks. IEEE communications magazine 40, 8 102-114. DOI: 10.1109/MCOM.2002.1024422. (2002).

[2] Chris Otto, Aleksandar Milenkovic, Corey Sanders : System architecture of a wireless body area sensor network for ubiquitous health monitoring. Journal of mobile multimedia 1, 4 (2006).

[3] N Alrajei, G Corser, H Fu, Y Zhu : "Energy Prediction Based Intrusion Detection in Wireless Sensor Networks". International Journal of Emerging Technology and Advanced Engineering 2014 .

[4] Marwa Salayma, Ahmed Al-Dubai and Imed Romdhani, Edinburgh Napier University Youssef Nasser, American University of Beirut. Wireless Body Area Network (WBAN): 'A survey on reliability, fault tolerance, and technologies coexistence'ACM 1544-3558. .2016.

[5] Karthic.k Raghunath and Rengarajan : "A Systematically Survey Investigation of Faults, Errors and Failures in Wireless Sensor Network “. International Journal of Advanced Computer Research (2013)

[6] Arunanshu Mahapatro.: Online fault detection and recovery in body sensor networks'. In Proceedings of the World Congress on Information and Communication Technologies (WICT), IEEE, 407412. DOI: 10.1109/WICT.2011.6141280. 2011

[7] B. Sharma, L. Golubchik, and R. Govindan "Sensor Faults: Detection Methods and Prevalence in Real-World Datasets,", ACM Transactions on Sensor Networks, vol. 6, no. 3, pp. 1-39, 2010.

[8] D. Giansanti, M. Dozza,:"Energetic assessment of trunk postural modifications induced by a wearable audio-biofeedback system," Medical engineering \& physics, vol. 31, no. 1, pp. 48-54, 2009.
[9] C. Wall, D. M. Wrisley "Vibrotactile tilt feedback improves dynamic gait index: a fall risk indicator in older adults, "Gait \& posture, vol. 30,no. 1. 16-21, 2009.

[10] C. Fritz and J. Poitevineau :"Influence of vibrotactile feedback on some perceptual features of violins,". The Journal of the Acoustical Society of America, vol. 136, no. 2, pp. 910-921, 2014.

[11] Fault Detection and Diagnosis in a Bayesian Network classifier incorporating probabilistic IFAC-Papers On Line 670-675,(2015).

[12] Mohamed Amine A to Sylvain Verron _ Abdessamad Kobi ."Fault Detection and Diagnosis in a Bayesian Network classifier incorporating probabilistic boundary': IFAC-Papers On Line 48-21 670-676, (2015).

[13] Adriana A. Alexandra: Using Expert Systems for Fault Detection and Diagnosis in Industrial Applications Research Institute for Informatics, Bucharest,

[14] Osman Salem, Alexey Guerassimov and Ahmed Mehaoua :Sensor Fault and Patient Anomaly Detection and Classification in Medical Wireless Sensor Networks. IEEE ICC 2013 - Selected Areas in Communications Symposium.

[15] Y. Yao, A. Sharma, L. Golubchik, and R. GovindaN: "Online Anomaly Detection for Sensor Systems: a Simple and Efficient Approach" n, Performance Evaluation, vol. 67, no. 11, pp. 10591075, 2010.

[16] B. Sharma, L. Golubchik, and R. Govindan :"Sensor Faults: Detection Methods and Prevalence in Real-World Datasets,", ACM Transactions on Sensor Networks, vol. 6, no. 3, pp. 1-39, 2010.

[17] A.Jabbari. W.Lang : "Applications of computer intelligence for sensor fault detection and isolation" . proceedings of world academy of science, engineering and technology vol 22. 2007.

[18] R.OGrady. M.Birattari : Fault detection in autonomous robots based on fault injection and learning' Autonomous robots, 2008.

[19] Model based fault detection and isolation using locally recurrent neural network. 'lecture notes on computer science vol.5097.2008.

[20] Yang Z X,Suzuki K.Shimada Y,SayayamaH.Fuzzy: "Fault diagnostic System based on fault tree analysis". IEEE APCCAS.1995:165-170.

[21] ZiJian Yang Beijing Graduate School."Survey of modern Fault Diagnosis methods in networks.", Chinese Academy of Sciences Beijing,

[22] Adriana A. Alexandra Research: "Using Expert Systems for Fault Detection and Diagnosis in Industrial Applications". Institute for Informatics, Bucharest.

[23] Yingying Wang ,Qiuju Li, Ming Chang, Hongwei Chen, Guohua Zang: International Conference on Advances in Computational Modelling and Simulation Research on Fault Diagnosis Expert System Based on the Neural Network and the Fault Tree Technology. Procedia Engineering $31 \quad$ (2012) 1206 - 1210 Elsevier Ltd.doi:10.1016.

[24] Sylvain Verron, Teodor Tiplica, Abdessamad Kobi: Fault Detection and Diagnosis in a Bayesian Network classifier incorporating probabilistic IFAC-Papers On Line 48-21 670-675., (2015)

[25] Mohamed Amine Atoui Sylvain Verron Abdessamad Kobi : Fault Detection and Diagnosis in a Bayesian Network classifier incorporating probabilistic boundary : IFAC-Papers On Line 48-21 670-676. (2015). 